\title{
Nivel de evidencia de los artículos publicados en la Revista Chilena de Cirugía. Evaluación del período 2007-2012*
}

\author{
Drs. JAVIER MORAGA C. ${ }^{1}$, RICARDO CARTES-VELÁSQUEZ ${ }^{2,3}$, SEBASTIÁN URRUTIA V. ${ }^{4}$, \\ CARLOS MANTEROLA D. ${ }^{1,2,4}$. GRUPO MINCIR (Metodología e Investigación en Cirugía).
}

1 Programa de Magíster en Ciencias Médicas, Mención Cirugía, Universidad de La Frontera. Temuco.

Programa de Doctorado en Ciencias Médicas, Universidad de La Frontera. Temuco.

Facultad de Odontología, Universidad de Concepción, Concepción.

4 Departamento de Cirugía y Traumatología. Facultad de Medicina, Universidad de La Frontera. Temuco. Chile.

\begin{abstract}
Level of evidence of articles published in Revista Chilena de Cirugía. Assessment of the period 2007-2011

Aim: To determine the level of evidence (LE) of articles published in Revista Chilena Cirugía (RCC) in the period 2007-2012 and their association with year of publication, subject area and university affiliation (UA). Material and Methods: Bibliometric study. An analysis of articles published between 2007 and 2012 in the RCC, applying the classification of the Oxford Centre for Evidence-Based Medicine (OCEBM) was performed. The sample is composed of all articles published in the period under study, which was feasible to apply the CEBM classification. The variables analyzed were LE, research design, year of publication, subject area and UA dichotomized (Yes/No). The LE of the items was determined by three independent investigators and discrepancies were resolved by consensus. We performed an exploratory analysis of the data and the results are presented using descriptive statistics. Results: Of the 688 articles published in the period, 452 meet the selection criteria. The most frequent LE was 4 and $2 b$ (91.4\% and 5.8\% respectively). The most frequent research designs were case report and retrospective case series ( $45.6 \%$ and $32.3 \%$ respectively). The subject areas that most articles provided were liver, biliary tract and pancreas; and esophagus, stomach, duodenum and small intestine (18.6\% and $18.1 \%$ respectively). The $76.5 \%$ of the articles presented UA. These had a higher proportion of articles with LE greater than 4 respect of those without UA (9.5\% vs $5.6 \%$ respectively). Conclusion: A significant proportion of articles published in RCC during the study period are of low LE.

Key words: "Bibliometrics" [MeSH], "Surgical procedures, operative" [MeSH], "Evidence-Based Medicine" [Mesh], Evidence levels.
\end{abstract}

*Recibido el 22 de noviembre de 2012 y aceptado para publicación el 4 de enero de 2013.

Los autores no refieren conflictos de interés.

Correspondencia: Drs. Javier Moraga C. y Carlos Manterola D. Manuel Montt 112, Oficina 408. Temuco, Chile. Javier.moragac@gmail.com,cmantero@ufro.cl 


\section{Resumen}

Objetivo: Determinar el nivel de evidencia (NE) de los artículos publicados en la Revista Chilena de Cirugía (RCC) en el período 2007-2012 y su asociación con año de publicación, área temática y filiación universitaria (FU). Material y Método: Estudio bibliométrico. Se realizó un análisis de los artículos publicados entre 2007 y 2012 en la RCC, aplicando la clasificación del Centro de Medicina Basada en Evidencia de Oxford (OCEBM). La muestra está compuesta por la totalidad de artículos publicados en el período en estudio, a los que fue factible aplicar la clasificación OCEBM. Las variables analizadas fueron NE, diseño de investigación, año de publicación, área temática y FU dicotomizada (Sí/No). El NE de los artículos fue determinado por 4 investigadores independientes, las discrepancias se resolvieron por consenso. Se realizó un análisis exploratorio de los datos y los resultados se presentan mediante estadística descriptiva. Resultados: De los 688 artículos publicados en el período, 452 cumplían los criterios de selección. Los NE más frecuentes fueron 4 y 2 b (91,4\% y 5,8\% respectivamente). Los diseños de investigación más prevalentes fueron reporte de casos y serie de casos retrospectiva (45,6\% y 32,3\% respectivamente). Las áreas temáticas que más artículos aportaron fueron hígado, vías biliares y páncreas; esófago, estómago, duodeno e intestino delgado (18,6\% y 18,1\% respectivamente). El 76,5\% de los artículos presentó FU, los que tenían mayor proporción de artículos de NE sobre 4 respecto de centros sin FU (9,5\% vs 5,6\% respectivamente). Conclusión: Una considerable proporción de los artículos publicados en la RCC en el período estudiado son de bajo NE.

Palabras clave: Bibliometría, cirugía, medicina basada en evidencia, niveles de evidencia.

\section{Introducción}

El conocimiento proveniente de los artículos científicos, no ofrece el mismo valor o impacto en la toma de decisiones en salud; por ello se requiere evaluar la calidad de la evidencia disponible. Los niveles de evidencia (NE) constituyen un sistema de jerarquización de ésta y corresponden a escalas alfanuméricas basadas solamente en el diseño del estudio $^{1,2}$.

El uso del concepto NE se remonta a la creación en 1976, de la Canadian Task Force on Preventive Health Care (CTFPHC), quienes generaron los NE y grados de recomendación para pacientes asintomáticos, indicando que procedimientos debían o no realizarse ${ }^{3}$. De esta forma, se tomó conciencia por primera vez acerca de la existencia de un orden jerárquico de la evidencia, en el que los resultados provenientes de estudios cuyos diseños de investigación presentaran mayor cantidad de sesgos no debían utilizarse en general para la toma de decisiones en clínica; especialmente si de cambio de conductas se tratase ${ }^{4}$.

En la actualidad, existen más de 100 sistemas distintos para la valoración de la evidencia ${ }^{1,5}$, como son: Canadian Task Force on Preventive Health Care (CTFPHC) $)^{3}$, National Institute for Health and Clinical Excellence (NICE) ${ }^{6}$, U.S. Preventive Services Task Force (USPSTF) ${ }^{7}$, Scottish Intercollegiate Guidelines Network (SIGN) ${ }^{8}$, Swedish Council on Technology Assessment in Health Care (SBU) ${ }^{9}$, Agencia d'Avaluació de Tecnología Médica (AATM) ${ }^{10}$, Niveles de evidencia de Sackett ${ }^{11,12}$, Oxford Centre for Evidence-Based Medicine (OCEBM) ${ }^{13}$, etc.

De todos los existentes, es posible que el más uti- lizado sea la propuesta del OCEBM, que se caracteriza por valorar la evidencia según el área temática o escenario clínico (tratamiento, prevención, etiología y daño; pronóstico e historia natural; diagnóstico; diagnóstico diferencial y estudios de prevalencia; y estudios económicos y análisis de decisión) y el tipo de estudio que involucra al problema en cuestión; de esta forma, jerarquiza la evidencia de acuerdo al mejor diseño para cada área temática.

El objetivo de este estudio, es determinar el nivel de evidencia (NE) de los artículos publicados en la Revista Chilena de Cirugía (RCC) en el período 2007-2012 y su asociación con año de publicación, área temática y filiación universitaria (FU).

\section{Material y Método}

Diseño: Estudio bibliométrico.

Población: Artículos publicados en la RCC entre los años 2007 y 2012 (ambos incluidos).

Criterios de selección: Se incluyeron artículos de investigación de todas las áreas quirúrgicas, publicados en la RCC en el período en estudio. Se excluyeron editoriales, artículos de revisión, cartas al editor y estudios in vitro; por lo que la muestra está compuesta por la totalidad de artículos publicados en este período, a los que fue posible aplicar la clasificación OCEBM ${ }^{13}$.

Variables en estudio: NE, diseño de investigación de los estudios, año de publicación, área temática y FU dicotomizada en Sí/No.

Maniobra: Se realizó una revisión de la totalidad de los números de la RCC publicados entre 2007 y 2012 (disponibles en http://cirujanosdechile.cl/ 
revista.htm). A los artículos encontrados se les aplicó los criterios de selección antes mencionados, posterior a lo cual se determinó NE de las publicaciones aplicando la clasificación $\mathrm{OCEBM}^{13}$ por 4 investigadores independientes (JM, RC-V, SU y $\mathrm{CM}$ ). Las discrepancias se resolvieron por consenso. Se asoció en NE con las otras variables en estudio.

Definiciones: Se definió como "área temática" a aquella área del conocimiento relacionada con la cirugía a la que adscribió el manuscrito. Para ello, los artículos fueron agrupados de la siguiente forma: Cirugía de cabeza-cuello y plástica; cirugía cardiovascular, cirugía hepatobiliar (hígado, vía biliar y páncreas), cirugía digestiva alta (esófago, estómago e intestino delgado); cirugía colorectal y misceláneas.

Se definió como "FU" a la relación de a lo menos uno de los autores del artículo con alguna universidad.

Plan de análisis: Se realizó un análisis exploratorio de los datos, posteriormente se utilizó estadística descriptiva de las variables con cálculo de medidas de tendencia central y dispersión. La tabulación y presentación de los datos se realizó con el software MS Excel 2007.

\section{Resultados}

Durante el período en estudio se publicaron 688 artículos con un promedio anual de 114,6 (Figura 1), de los cuales 452 cumplían los criterios de selección.

Los NE más frecuentemente hallados fueron: 4 y 2 b con un 91,4\% y 5,8\% respectivamente (Tabla 1). Mientras que los diseños más prevalentes fueron: reporte de casos, series de casos retrospectivas y prospectivas, con un $45,6 \% ; 32,3 \%$ y $12,0 \%$ respectivamente (Tabla 2 ).

El año que presentó mayor cantidad de artículos publicados fue 2010; sin embargo, el mejor NE fue en 2011 con 9 artículos de NE mayor a 4; lo que representa un $11,7 \%$ del total de artículos publicados durante ese año. El NE de los artículos por año se observa en la Tabla 3.

El área temática que mayor cantidad de artículos aportó durante el período en estudio, fue cirugía hepatobiliar con 84 artículos (18,6\%); sin embargo, el área temática de cirugía digestiva alta fue la que
Tabla 1. Distribución de los artículos publicados en RCC según NE (2007-2012)

\begin{tabular}{|ccc|}
\hline Nivel de evidencia & n artículos & $\%$ \\
\hline $1 \mathrm{~b}$ & 5 & 1,1 \\
$2 \mathrm{a}$ & 1 & 0,2 \\
\hline $2 \mathrm{~b}$ & 26 & 5,8 \\
\hline $2 \mathrm{c}$ & 4 & 0,9 \\
\hline $3 \mathrm{a}$ & 2 & 0,4 \\
\hline $3 \mathrm{~b}$ & 1 & 0,2 \\
\hline 4 & 413 & 91,4 \\
\hline Total & 385 & 100 \\
\hline
\end{tabular}

Tabla 2. Distribución de los artículos publicados en RCC según diseño de investigación (2007-2012)

\begin{tabular}{|lrc|}
\hline Diseño & n & \% \\
RS de estudios de cohortes & 1 & 0,2 \\
Estudios de cohortes prospectiva & 11 & 2,4 \\
EC de baja calidad & 2 & 0,4 \\
RS no de EC & 3 & 0,7 \\
Estudios de casos y controles & 4 & 0,9 \\
Estudios de corte transversal & 5 & 1,1 \\
Estudios de cohortes retrospectivas & 20 & 4,4 \\
Series de casos prospectivas & 54 & 12,0 \\
Series de casos retrospectivas & 146 & 32,3 \\
Reporte de casos & 206 & 45,6 \\
Total & 452 & 100,0 \\
\hline
\end{tabular}

RS: Revisiones sistemáticas de la literatura. EC: Ensayos clínicos con asignación aleatoria.

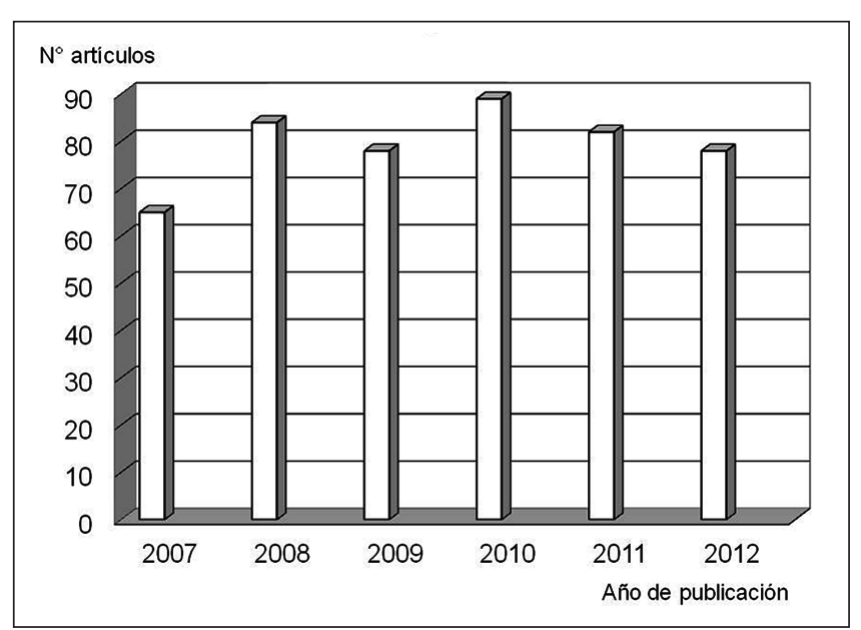

Figura 1. Distribución de los artículos analizados según año de publicación. 
presentó el mejor NE con 12 artículos de NE mayor a 4 correspondientes al 14,6\% de los artículos de esta área temática. Ver Tabla 4.

El $76,5 \%$ de los artículos presentó $\mathrm{FU}$, los que tenían mayor proporción de artículos con NE sobre 4 respecto de centros no universitarios $(9,5 \%$ vs $5,6 \%$ respectivamente). Los centros universitarios cuyos artículos presentaron mejor NE fueron la Universidad de La Frontera y la Universidad Austral de Chile; con 47,1\% y $16,7 \%$ de sus artículos con NE mayor a 4 respectivamente. El NE de los artículos de las 5 universidades con más publicaciones se observa en la Tabla 5.

\section{Discusión}

La valoración de la evidencia, en un inicio, se encontró marcada por la necesidad de la comunidad científica de sistematizar el conocimiento; dando de esta forma, respuestas a las preguntas surgidas de la práctica clínica cotidiana. Esto, llevó a cambiar la forma de tomar las decisiones con respecto a políticas públicas de salud, donde sólo el conocimiento "más válido" debía ser utilizado para tales fines ${ }^{1-3}$. En tal sentido, la actividad investigativa debe siempre propender al más alto nivel y evitar, dentro de lo posible, invertir tiempo y recursos en la realización de investigaciones que tienen un aporte marginal, que poco sustento brinde a la práctica diaria.

De los resultados del presente estudio, destaca que el NE de los artículos publicados en la RCC es bajo. Fenómeno transversal en las disciplinas quirúrgicas a nivel mundial ${ }^{14}$; sin embargo, la proporción de investigaciones meramente descriptivas es aún mayor en la RCC, aunque comparable a otras publicaciones quirúrgicas en español ${ }^{15}$. Escapa de los objetivos de esta investigación, determinar el origen de tal situación, que muy probablemente es multifactorial, y necesitan ser dilucidadas en futuras investigaciones.

Uno de los posibles efectos de este bajo $\mathrm{NE}$ es el bajo impacto y visibilidad que aún presentan los artículos publicados en la $\mathrm{RCC}^{\mathbf{1 6}}$, concordante con la producción científica de la cirugía chilena ${ }^{17}$; por lo que en miras a mejorar estos indicadores, una de las posibles estrategias a implementar, sea el desarrollar investigaciones con NE 3
Tabla 3. Distribución de los artículos publicados en RCC según NE y año de publicación (2007-2012)

\begin{tabular}{|ccccccc|}
\hline Año & $\begin{array}{c}\text { NE } \\
\mathbf{1 , 2 , 3}\end{array}$ & $\mathbf{\%}$ & $\begin{array}{c}\text { NE } \\
\mathbf{4}\end{array}$ & $\mathbf{\%}$ & Total & $\mathbf{\%}$ \\
\hline 2007 & 5 & 8,1 & 57 & 91,9 & 62 & 16,1 \\
\hline 2008 & 7 & 8,6 & 74 & 91,4 & 81 & 21,0 \\
\hline 2009 & 5 & 6,5 & 72 & 93,5 & 77 & 20,0 \\
\hline 2010 & 7 & 8,0 & 81 & 92,0 & 88 & 22,9 \\
\hline 2011 & 9 & 11,7 & 68 & 88,3 & 77 & 20,0 \\
\hline 2012 & 6 & 9,0 & 61 & 91,0 & 67 & 14,8 \\
\hline Total & 39 & 8,6 & 413 & 91,4 & 452 & 100 \\
\hline
\end{tabular}

Tabla 4. Distribución de los artículos publicados en RCC según NE y área temática (2007-2012)

\begin{tabular}{|c|c|c|c|c|c|c|}
\hline Área Temática & $\begin{array}{c}\mathrm{NE} \\
1,2,3\end{array}$ & $\%$ & $\begin{array}{c}\text { NE } \\
4\end{array}$ & $\%$ & Total & $\%$ \\
\hline C. Hepatobiliar & 11 & 13,1 & 73 & 86,9 & 84 & 18,6 \\
\hline C. Digestiva alta & 12 & 14,6 & 70 & 85,4 & 82 & 18,1 \\
\hline C. Colorectal & 5 & 8,3 & 55 & 91,7 & 60 & 13,3 \\
\hline $\begin{array}{l}\text { C. Cabeza-cuello y } \\
\text { plástica }\end{array}$ & 2 & 3,1 & 62 & 96,9 & 64 & 14,2 \\
\hline C. Cardiovascular & 1 & 3,4 & 28 & 96,5 & 29 & 6,4 \\
\hline Misceláneas & 8 & 6,0 & 125 & 94,0 & 133 & 29,4 \\
\hline Total & 39 & 8,6 & 413 & 91,4 & 452 & 100,0 \\
\hline
\end{tabular}

Tabla 5. NE de los artículos de las universidades con mayor número de publicaciones en RCC (2007-2012)

\begin{tabular}{|c|c|c|c|c|c|c|}
\hline Universidad & $\begin{array}{c}\mathrm{NE} \\
1,2,3\end{array}$ & $\%$ & $\begin{array}{c}\text { NE } \\
4\end{array}$ & $\%$ & Total & $\%$ \\
\hline Universidad de Chile & 8 & 6,3 & 119 & 93,7 & 127 & 36,8 \\
\hline $\begin{array}{l}\text { Pontifica Universidad } \\
\text { Católica de Chile }\end{array}$ & 2 & 3,1 & 63 & 96,9 & 65 & 18,8 \\
\hline $\begin{array}{l}\text { Universidad de } \\
\text { La Frontera }\end{array}$ & 16 & 47,1 & 18 & 52,9 & 34 & 9,9 \\
\hline $\begin{array}{l}\text { Universidad de } \\
\text { Concepción }\end{array}$ & 0 & 0,0 & 18 & 100 & 18 & 5,2 \\
\hline $\begin{array}{l}\text { Universidad Austral } \\
\text { de Chile }\end{array}$ & 3 & 16,7 & 15 & 83,3 & 18 & 5,2 \\
\hline Otras & 4 & 4,8 & 79 & 95,2 & 83 & 24,1 \\
\hline Total & 51 & 14,8 & 294 & 85,2 & 345 & 100,0 \\
\hline
\end{tabular}

o mejores; debemos recordar que durante el año 2009 la RCC fue indizada en la base de datos Science Citation Index Expanded de Thompson Reuters (ISI) ${ }^{18}$, ubicándose en el cuarto cuartil según el Journal Citation Report de 2011, con un factor de impacto 0,184; lejos de revistas que generan impacto en 
la toma de decisiones en cirugía como Annals of Surgery o British Journal of Surgery cuyos FI son de 7,492 y 4,606 respectivamente ${ }^{19}$.

Al valorar el NE de los artículos por año, se observa que el número de artículos con NE mayor a 4 se mantiene relativamente constante entre $8 \%$ y $12 \%$ del total de artículos por año; lo que puede ser preocupante no sólo por la gran cantidad de artículos NE 4; sino también, porque al desglosar aquellos con mejor evidencia observamos que sólo $5(1,3 \%$ del total) era de NE 1; lo que representa menos de una publicación de este tipo por año.

Destaca también en los resultados que aproximadamente el $50 \%$ de las publicaciones provienen de 3 áreas de la cirugía (hepatobiliar; digestiva alta y colorrectal); y que a su vez sean las dos primeras las que presentan proporcionalmente mayor proporción de artículos con mejor NE.

Otro hecho interesante en este análisis es que más de la mitad de los artículos publicados provienen de 2 comunidades universitarias, situación que suscita algunos comentarios. El primero en relación a la gran capacidad productiva que presentan estas instituciones; y el segundo, que más del $95 \%$ de estas son de bajo NE (tipo 4), afectando de esta forma el NE global de la RCC. Esta situación podría ser explicada por diferentes motivos, como por ejemplo que aquellos artículos con mejor NE sean enviados a publicación a revistas de mayor FI, siendo una forma de presentación del denominado "efecto mateo" 20,21 .

Todo lo anterior, corresponde sólo a una mirada parcial al problema, pues los NE no implican necesariamente calidad metodológica (CM); sin embargo, en un estudio anterior de nuestro grupo de trabajo, realizado a nuestra revista, se verificó una asociación entre ambos conceptos ${ }^{22}$.

Otro de los resultados relevantes, no tan sólo para el "diagnóstico" de la situación, sino también para un potencial "plan de mejora"; es que los artículos con FU presentan mejor NE; situación que parece lógica si pensamos que son las universidades las instituciones llamadas a generar conocimiento. Por ende, una de las posibles estrategias para mejorar el NE, podrían ser la formación de capital humano avanzado en programas de Magíster académico y Doctorado; la incorporación a las unidades académicas, de recurso humano con formación en investigación clínica; y la asociación de estas con centros sin FU que deseen realizar investigación.

Este estudio ha pretendido valorar de forma objetiva el perfil de la evidencia publicada en la RCC entre los años 2007 y 2012, con la intención de ser un aporte y punto de partida para reflexionar y tomar acciones tendientes a mejorar las situaciones que lo requieran, en el corto, mediano y largo plazo.

\section{Referencias}

1. Manterola C, Zavando D. Como interpretar los "Niveles de Evidencia" en los diferentes escenarios clínicos. Rev Chil Cir. 2009;61:582-95.

2. Burgos M, Manterola C, Sanhueza A. Diseño de una escala para evaluar calidad metodológica de estudios de pruebas diagnósticas. Estudio piloto. Rev Chil Cir. 2011;63:493-7.

3. Canadian Task Force on Preventive Health Care. New grades for recommendations from the Canadian Task Force on Preventive Health Care. CMAJ 2003;169:20720.

4. UpShur RE. Are all evidence-based practice alike? Problems in the ranking of evidence. CMAJ 2003;169:672-3.

5. West S, King V, Carey TS, Lohr KN, McKoy N, Sutton SF, et al. Systems to Rate the Strength of Scientific Evidence. Health Services/Technology Assessment Text, National Library of Medicine. AHRQ Publication No. 02-E016, 2002. Disponible en: http://www.ncbi.nlm.nih. gov/books/bv.fcgi?rid=hstat1.chapter.70996. Visitado el 18 de octubre de 2012.

6. National Institute for Health and Clinical Excellence (NICE). The guidelines manual 2009, Disponible en: http://www.nice.org.uk. Visitado el 15 de octubre de 2012.

7. Task Force Ratings, Guide to Clinical Preventive Services, Second Edition, Disponible en: http://odphp. osophs.dhhs.gov/pubs/guidecps. Visitado el 20 de diciembre de 2012.

8. Scottish Intercollegiate Guidelines Network (SIGN). SIGN 50, A guideline developer's handbook, revised edition 2008. Disponible en: http://www.sign.ac.uk/pdf/ sign50.pdf. Visitado el 20 de diciembre de 2012.

9. Goodman C. Literature searching and evidence interpretation for assessing health care practices. Stockholm, Sweden: Swedish Council on Technology Assessment in Health Care, 1993:16-32.

10. Jovell A, Navarro-Rubio M. Evaluación de la evidencia científica. Med Clin. (Barc) 1995;105:740-3.

11. Sackett DL, Wennberg JE. Choosing the best research design for each question. BMJ 1997; 315:1636.

12. Sackett DL. Rules of evidence and clinical recommendations on use of antithrombotic agents. Chest 1986;89(2 suppl):2S-3S.

13. Oxford Centre for Evidence-based Medicine (CEBM). Centre for Evidence Based Medicine - Levels of Evidence (March 2009). Disponible en: http://www.cebm.net/ index.aspx?o=1025. Visitado el 20 de diciembre de 2012.

14. Manterola C, Pineda V, Vial M, Losada H. What is the methodologic quality of human therapy studies in ISI surgical publications? Ann Surg. 2006;244:827-32.

15. Ibáñez V, Modesto V, Lluna J, Hernández E. Nivel de evidencia científica en la cirugía pediátrica española. Cir Pediatr. 2005;18:93-8.

16. Cartes-Velásquez R, Moraga J, Aravena P, Manterola C. 
Impacto y visibilidad de la revista chilena de cirugía tras su indización en SciELO e ISI. Análisis bibliométrico. Rev Chil Cir. 2012;64:511-5.

17. Moraga J, Cartes-Velásquez R, Manterola C, Urrutia S. Publicaciones de autores chilenos en revistas quirúrgicas durante los últimos diez años. Rev Chil Cir. 2012;64:447-51.

18. Arribalzaga E. Incorporación al ISI de la Revista Chilena de Cirugía. Rev Chil Cir. 2009;61:313.

19. Thomson-Reuters. Journal Citation Report 2001. Dispo- nible en http://admin-apps.webofknowledge.com/JCR/ JCR. Visitado el 15 de enero de 2013.

20. Jiménez J. El efecto mateo: un concepto psicológico. Papeles del Psicólogo 2009;30:145-54.

21. Bonitz M. Ten years Matthew effect for countries. Scientometrics 2005;64:375-79.

22. Pineda V, Manterola C, Vial M, Losada H. ¿Cuál es la calidad metodológica de los artículos referentes a terapia publicados en la Revista Chilena de Cirugía? Rev Chil Cir. 2005;57:500-7. 